\title{
Diversidad de usuarios en las agencias bancarias: Un análisis de clases latentes, municipio Libertador, estado Mérida, Venezuela
}

\author{
Marysela Coromoto Morillo Moreno | morillom@ula.ve \\ Universidad de Los Andes, Venezuela \\ Milángela Del Carmen Morillo Moreno | morimorenca@hotmail.com \\ Universidad de Los Andes, Venezuela
}

Malinda Del Valle Coa Ravelo | malinda@ula.ve

Universidad de Los Andes, Venezuela

Recibido en: 26 de septiembre de 2014

Aceptado en: 17 de abril de 2015

\section{RESUMEN}

Para determinar la existencia de distintos grupos de usuarios de los servicios personales de las agencias bancarias a partir de características demográficas, psicográficas y de comportamiento, se diseñó una investigación de campo, basada en un muestreo aleatorio, en el municipio Libertador del estado Mérida, Venezuela. Como hallazgo, un análisis estadístico de clases latentes destaca la existencia de tres clases de usuarios; la clase que agrupa el mayor número de usuarios se caracteriza por tener una frecuencia de uso y percepción moderada sobre la calidad. La identificación de tales grupos precede el diseño de prácticas de marketing para cada segmento, especialmente para el manejo de tiempos de espera, calidad y recuperación del servicio.

Palabras clave: calidad, servicios bancarios y segmentación de mercados.

\section{ABSTRACT}

To determine the existence of different groups of users of personal services of the banking agencies, based on demographic, psychographic and behavioral characteristics, field research was designed, based on random sampling, in the Libertador municipality of Merida state, Venezuela. As finding, a statistical analysis of latent classes highlights the existence of three classes of users; the kind that brings the largest number of users is characterized by a moderate frequency of use and perception over quality. The identification of such groups precedes the design of marketing practices for each segment, particularly for handling waiting times, service quality recovery.

Keywords: quality, banking and market segmentation. 
La dinámica competitiva de las organizaciones se centra cada vez más en la prestación de servicios antes, durante y después de la venta de los productos tangibles convirtiéndose esta prestación en uno de los elementos estratégicos claves de la competitividad. En el sector bancario los servicios personales persisten, pues aún cuando son muchos los encuentros que se tienen con el cliente a distancia (a través de teléfono, cajeros automáticos e internet), los encuentros personales continúan siendo necesarios y generadores de muchas experiencias. El servicio personal es un tipo de servicio al cliente, que Zeithaml y Bitner (2002) lo definen como uno de los encuentros en el cual se establece relación directa, cara a cara, con el cliente a través de un empleado denominado personal de primera línea o de vanguardia. Actualmente las instituciones bancarias, según Rodríguez, Padrón y Olivera (2004), se encuentran bajo elevadas presiones competitivas, por lo que han diseñado estrategias dirigidas a nuevos mercados dominados por instituciones no bancarias. Igualmente, según Berry, Bennet y Brown (1989), la banca reconoce que la cartera de productos ofrecidos por diferentes instituciones bancarias coinciden, pero aunque cada institución las adapta a sus propias estrategias para diferenciarse, esta no es lo única alternativa; existe otra muy válida y reconocida como es la calidad y la diferenciación que puede establecerse en el servicio personal prestado.

De acuerdo a Alcaide y Soriano (2006), la relación cliente-banco es compleja; la misma no puede limitarse a la simple gestión de contacto y buen trato en relaciones interpersonales, sino que requiere de mayores esfuerzos y enfoques amplios y profundos. El encuentro o contacto personal es solo una mínima parte o el resultado del esfuerzo. Según los autores, el esfuerzo debe comenzar por realizar una correcta segmentación de sus clientes, con el fin de conocer con precisión objetivos y necesidades de cada tipo de cliente y determinar cuáles son los beneficios que procura en la relación; además, se podrán atenuar los costos mediante el ofrecimiento efectivo de los productos y servicios, y por ende elevar los niveles de satisfacción de los clientes. También Berry et al. (1989) indican que antes del buen servicio personal la base para la diferenciación bancaria es la segmentación, como la "identificación de grupos de clientes que serán su 
blanco principal, y después el desarrollo de combinaciones mercantiles adaptadas para cubrir sus necesidades" (p. 8).

A nivel internacional, de acuerdo a la Cátedra de Contabilidad Bancaria de la Universidad de la República de Uruguay (2013), en la segmentación del mercado financiero existen dos grandes mercados extensos y heterogéneos: el mercado minorista (economías familiares) y el mercado mayorista o corporativo (organizaciones públicas y privadas). En el sector financiero, según Abad y Alegría (1984), la segmentación es un concepto relativamente nuevo, de la década de los años 70; desde entonces, se han identificado de forma progresiva nuevos segmentos de interés: la banca para empresas (pequeñas, medianas y grandes) y la banca para particulares. En España, los primeros bancos en aplicar la segmentación de mercados fueron el Banco Bilbao y el Banco de Vizcaya, estos establecieron una nueva estrategia comercial. En la actualidad, la segmentación más empleada es la múltiple o diferenciada, centrándose en varios segmentos, y la menos usada es la segmentación masiva, en la que se lanzan productos al mercado indiscriminadamente (Bello, 2012).

En Venezuela, según Romero y Artigas (2008), la banca segmenta su mercado según la Federación Latinoamericana de Bancos (Felaban), es decir, "con base a la estructura de los depósitos de los clientes” (p. 84), dejando de lado características propias del consumidor que podrían beneficiarlos y diferenciarlos. De acuerdo a la Felaban (2008), el objeto de la segmentación del mercado es "analizar las operaciones de un cliente para definir si son ... sospechosas” (p. 81). Esta plantea la comparación entre clientes pertenecientes a un mismo segmento, para deducir lo inusual de sus operaciones e identificar algunos delitos financieros.

Para Romero y Artigas (2008, p. 84), esta segmentación es producto del "control estatal en el manejo de operaciones"; por ello, no puede ser tomada efectivamente como elemento diferenciador en el sector financiero. Romero y Artigas (2008) exponen que, la banca venezolana segmenta en cierta medida de forma geográfica y demográfica tomando en cuenta las localizaciones de las sucursales y los elementos cuantitativos y cualitativos como edad, posición social, nivel de ingresos u operaciones realizadas. Estos criterios 
reflejan la práctica de la "segmentación demográfica orientada al ámbito financiero” (p. 85). Por ello, según Romero y Artigas (2008), las instituciones financieras venezolanas deben establecer un enfoque relacional e introducir elementos diferenciadores, mediante una adecuada segmentación de mercado que incluya la ponderación de todos los elementos posibles de segmentación, y así conocer concretamente el mercado meta, haciendo un uso eficiente de su base de datos.

Bajo esta óptica en el contexto bancario y considerando que, para Rondán, Villarejo y Sánchez (2007), el análisis de clases latentes permite establecer agrupaciones de comportamientos basados en relaciones a priori difícilmente detectables, en esta investigación se intentan sentar las bases para iniciar el desarrollo de estrategias de segmentación mediante la determinación de grupos homogéneos de usuarios, a partir de sus características, como paso previo a la selección y el posicionamiento de los servicios bancarios en los segmentos adecuados.

\section{FUNDAMENTOS TEÓRICOS}

Para Martín, Manera y Pérez (1998), Mullins, Walner, Boyd y Larréché (2007) y Stanton, Etzel y Walker (2007), el proceso de segmentación es la división del mercado total de un bien o servicio en varios subconjuntos o grupos menores de clientes con características homogéneas, que los lleva a responder de forma semejante ante la oferta de un producto o programa de marketing; esto lo hace merecedor de ser constituido como un mercado meta para el diseño de una mezcla de mercadotecnia. Entre las ventajas destacan el mejor aprovechamiento de los recursos escasos así como una satisfacción específica y cuidadosa de las necesidades del cliente; también la segmentación es el primer paso para el posicionamiento en el mercado, creando una imagen única ante el consumidor mediante el diseño y las ofertas de productos para el segmento seleccionado.

De acuerdo a Stanton et al. (2007), partiendo que un grupo de clientes que comparte un deseo distinguible del resto del mercado 
es un segmento, un primer paso para la segmentación es la determinación de las características que comparten dichos usuarios con deseos similares, de forma mensurables y asequibles. Un segundo paso es determinar el tamaño de los segmentos y el grado en que se está satisfaciendo, es decir, cuánta demanda representan lo que a su vez expresa su atractivo.

Según Kotler y Keller (2006) y Stanton et al. (2007), existen criterios de segmentación geográficos, demográficos, psicográficos y de comportamiento o conductuales. Los geográficos se vinculan a las localizaciones donde el cliente reside o se desempeña, que suelen dictaminar sus deseos. Los demográficos están determinados por edad, sexo, ingresos y educación, lo cual guarda estrecha relación con los niveles de demanda. Los psicográficos se relacionan con la personalidad, la forma de pensar y sentir, el estilo de vida, los valores, la sensibilidad ante la calidad y otros criterios. Finalmente, la segmentación conductual se basa en actitudes o respuestas hacia el producto, los beneficios buscados, los términos y la tasa de uso, y las promociones, entre otras.

En el ámbito bancario, Minhas y Jacobs (1996), afirman que las características típicas para segmentar los mercados tienen escaso poder para predecir el comportamiento de compra de los usuarios; en su lugar proponen criterios relacionados con la compra de clientes. De esta manera las instituciones financieras pueden comercializar eficaz y eficientemente sus servicios, con mayores beneficios y penetrando nuevos mercados. Bravo, Montaner y Pina (2009), presentan una recopilación de las principales variables utilizadas para segmentar los usuarios de los servicios bancarios; y mencionan no solo variables tradicionales, sino también relacionadas con el comportamiento de compra (sexo, edad, ingresos, nivel de estudios, situación laboral, estado civil, etnias, estilos de vida, lealtad, rentabilidad para el banco de los clientes, número y servicios contratados, participación del cliente, confianza e incertidumbre, beneficios buscados).

Como un aporte en la segmentación bancaria, Bravo et al. (2009) en su investigación con cinco entidades financieras españolas (Santander, BBVA y tres cajas de ahorros: La Caixa, Ibercaja y Caja 
de ahorros de la Inmaculada) hallaron diferencias entre usuarios, según variables sociodemográficas, a la hora de valorar la imagen corporativa de la banca. En su trabajo evidencian baja valoración en los pequeños usuarios, en los grupos de clientes endeudados y con menor nivel de satisfacción, y a partir de allí recomiendan analizar si la solución de ofrecer una mayor información sobre los servicios podría mejorar la imagen.

En el caso del estudio realizado por Chau y Ngai (2010) sobre las percepciones, las actitudes y los comportamientos de los usuarios de la banca por internet, se hallaron diferencias importantes entre las actitudes e intenciones conductuales de los jóvenes (de 16 a 29 años de edad), estudiantes universitarios y otros grupos de usuarios. Ello implica la necesidad de desarrollar persistentemente la calidad del sitio web, en pro de los beneficios a largo plazo del banco y la retención de clientes jóvenes, dadas sus elevadas actitudes e intenciones conductuales hacia el uso de los servicios electrónicos y su rentabilidad futura. Stafford (1996), al determinar los elementos de la calidad del servicio, halló que segmentos de mercado agrupados por variables demográficas perciben a los elementos de la calidad del servicio con distintos grados de importancia; la calidad del servicio parece más importante para las mujeres e insiste que los proveedores del servicio deben comprender a sus clientes y lo que es importante para ellos en relación con la calidad.

Posterior a la determinación de las variables para la segmentación, de acuerdo a Martín et al. (1998), en la búsqueda de la homogeneidad interna y heterogeneidad entre grupos, suelen intervenir varios procedimientos estadísticos cualitativos o cuantitativos, (métodos de clusterización, análisis de varianza, análisis discriminante, análisis factorial de correspondencia y otros) de elevado valor en los servicios financieros, en los que la relación con el cliente es un factor clave para el éxito (Data, Decisions and Language).

Considerando que, en esta investigación destaca como una de las características conductuales del usuario su percepción de la calidad del servicio, constructo indefinido e interpretado desde distintos puntos de vista a lo largo del tiempo (Setó, 2005), a continuación se exponen los fundamentos teóricos de la calidad del servicio. 
Según Garvín (1988) la gestión de la calidad ha evolucionado desde la etapa de la simple inspección, pasando por el control estadístico y el aseguramiento de la misma, hasta alcanzar la calidad desde el punto de vista del cliente, como estrategia competitiva. En la actualidad, de acuerdo a Grönroos (1984) y Reeves y Bednar (1994), la calidad en los servicios es el grado de conformidad de los atributos y características de un servicio respecto a las expectativas del cliente o de la satisfacción de las expectativas del mismo. Es el resultado de la evaluación realizada por el usuario mediante la comparación entre lo que los clientes perciben de la prestación del servicio y lo que estos esperaban. Una vez comprado y utilizado el servicio, los resultados son confrontados con las expectativas y si se produce coincidencia entre ambos, tiene lugar la confirmación; pero, si existen discrepancias entre los resultados y las expectativas, lo que se produce entonces es la disconfirmación. Así la calidad fue concebida por Zeithaml, Parasuraman y Berry (1985, 1990, 1993a), Cantú (2006), Kotler y Keller (2006), Hoffman y Bateson (2002), Zeithaml y Bitner (2002), Ginebra y Arana (1999), Pride y Ferrell (1997) y Lele y Sheth (1987).

El análisis y la evaluación de la calidad del servicio están dados por una serie de modelos conceptuales e instrumentos. Según Brogowicz et al. (1990), una primera aproximación a la calidad de servicio puede estructurarse a partir de dos grandes escuelas de conocimiento: la escuela norteamericana y la norteuropea o nórdica. La escuela norteamericana, liderada por Zeithaml et al. (1985, 1988), presenta como modelos las cinco dimensiones y las diferencias entre expectativas y percepciones, derivándose otros modelos como el jerárquico multidimensional y el integral de brechas sobre la calidad, así como instrumentos diseñados como escalas de medida de la calidad y sus derivadas. En esta investigación se utilizó la escala de Servqual (acrónimo de service quality), para medir la calidad del servicio experimentado por el usuario, como variable conductual. A continuación se describe el origen y funcionamiento de dicha escala.

De acuerdo a la revisión practicada en los trabajos de sus creadores (Zeithaml, Parasuraman, \& Berry, 1985, 1988, 1993a), Servqual es un instrumento de diagnóstico genérico que puede ser aplicado 
en cualquier organización, para descubrir fortalezas y debilidades en la prestación del servicio, basándose en las cinco dimensiones de la calidad del servicio. En un primer momento, para la década de los años 80, los investigadores Valarie Zeithaml, A. Parasuraman y Leonard Berry realizaron un estudio exploratorio entre consumidores, a través de 12 sesiones de grupo, compuesto por tres grupos para cuatro sectores de servicios (banca minorista, tarjeta de crédito, corredores de valores y reparación y mantenimiento de aparatos). En dichas sesiones los clientes hablaron sobre las expectativas, experiencias, prioridades, los atributos del servicio y las personas que suministraron el servicio. A partir de allí se detectaron patrones consistentes en las respuestas de los cuatro grupos, que permitieron ver cómo los clientes definen y evalúan la calidad de los servicios, los factores que influyen en las expectativas del usuario y la existencia de las diez dimensiones clásicas de la calidad del servicio (Zeithaml et al., 1993b). Fue así como identificaron diez criterios o dimensiones que reflejan la calidad de un servicio; posteriormente fueron agrupadas en cinco dimensiones: tangibilidad, empatía, seguridad o garantía, respuesta y confiabilidad, como elementos prácticos y resumidos. Combinando el modelo de las cinco dimensiones y de diferencias entre expectativas y percepciones del usuario, los mismos investigadores abordaron la fase cuantitativa de la investigación para desarrollar un instrumento para medir la calidad del servicio (Servqual) (Zeithaml et al., 1993a).

Específicamente, Servqual consta de 22 ítems que intentan recolectar las expectativas sobre un excelente servicio, seguidos de otros 22 ítems que recolectan las percepciones sobre las mismas dimensiones, en un servicio específico, para un total de 44 puntos. Las puntuaciones de medida de cada dimensión, están comprendidas en una escala desde lo totalmente de acuerdo, hasta lo totalmente en desacuerdo. Una vez recolectados los datos, las diferencias existentes entre las expectativas y percepciones se constituyen en brechas del servicio, las cuales al ser calculadas cuantitativamente se les denomina puntuaciones de Servqual; mientras más bajas sean las percepciones respecto a las expectativas, más baja será la evaluación de la calidad del servicio. 


\section{Objetivos e hipótesis de La INVESTIGACIÓN}

De acuerdo a Kotler y Keller (2006), los pasos hacia la mercadotecnia orientada al mercado meta se inician con la identificación de las bases para la segmentación y el desarrollo de perfiles de segmentos resultantes; por su parte, las investigaciones de Chau y Ngai (2010), Stafford (1996), Bravo et al. (2009) y Minhas y Jacobs (1996) recomiendan continuar investigando sobre la segmentación de mercado a partir de variadas bases o criterios, en distintas realidades y amplios contextos. Considerando esto, posterior a la revisión teórica se plantea como objetivo general determinar la existencia de distintos grupos de usuarios de los servicios personales de la agencias bancarias del municipio Libertador del estado Mérida, Venezuela, a partir de características demográficas, psicográficas y de respuestas del usuario o de comportamiento de uso (conductuales).

Como objetivos específicos se plantean:

1) Determinar los grupos homogéneos de usuarios que pueden distinguirse, a partir de variables demográficas, psicográficas y de comportamiento de uso.

2) Identificar los patrones o características determinantes que permiten distinguir tipologías o grupos de usuarios de las agencias bancarias en estudio.

3) Estimar el tamaño y las características de cada grupo de usuarios de las agencias bancarias.

Se formularon las siguientes hipótesis:

$\mathrm{H}_{1}$. Existen grupos homogéneos de usuarios en las agencias bancarias del municipio Libertador del estado Mérida.

$\mathrm{H}_{2}$. Las tipologías de usuarios de las agencias bancarias estudiadas atienden variables demográficas, psicográficas y de comportamiento de los usuarios.

Es importante señalar, que esta investigación fue delimitada geográficamente en las agencias bancarias ubicadas en el municipio Libertador del estado Mérida, Venezuela, dado que según Morillo 
(2010), es el municipio que posee la mayor densidad poblacional del Estado (27,95\%), con un incremento el 50,5 a 76,1 hab/km2 en los últimos 20 años; esto explica en gran medida el desarrollo de diversas actividades económicas basadas en diversos servicios, incluidos los financieros. Por otra parte, dada la necesidad de diversificación económica venezolana, el estado Mérida a su vez representa una de las regiones económicas más importantes de Venezuela, por basar su economía en la actividad agrícola acompañada de múltiples servicios (comerciales, turísticos y educativos) públicos y privados, y por su gran potencial al ocupar una superficie de $11.300 \mathrm{~km} 2$ ( $1,23 \%$ del territorio nacional), concentrando apenas cerca del 3,0\% de la población del País, debido a las áreas montañosas.

\section{Metodología}

Se diseñó una investigación con un enfoque cuantitativo, de tipo documental y de campo, basada en un muestreo probabilístico en el cual se recolectaron datos de los usuarios de las agencias bancarias de las instituciones financieras ubicadas en el municipio Libertador del estado Mérida, por ser este el municipio de la entidad que agrupa la mayor cantidad de agencias bancarias de la mayoría de entidades financieras, públicas y privadas de Venezuela.

Se definió como población objeto de estudio a los usuarios de las agencias bancarias pertenecientes a las instituciones financieras ubicadas en el municipio Libertador del estado Mérida, Venezuela; dichas agencias aparecen en el directorio elaborado de la Superintendencia Nacional de Bancos y Otras Instituciones Financieras de la República Bolivariana de Venezuela (2006), conformando un total de 40 agencias pertenecientes a los diferentes subsistemas del sistema financiero venezolano (banca universal y comercial).

Debido a que el investigador no pudo determinar el tamaño de la población objeto de estudio ${ }^{1}$ y con el propósito de generalizar los resultados a la población de usuarios, se seleccionó una muestra basada en el criterio de la varianza máxima como un muestreo probabilístico para una población infinita (Hurtado, 2000). Fue así como, con un nivel de confianza del 95\%, un 
error de estimación $\left(e^{2}\right)$ del $5 \%$ y unas proporciones de éxitos (p) y de fracasos (q) iguales a 0,5, se estudió una muestra de 384 usuarios $\left(n=\frac{\mathrm{Z}^{2} \mathrm{a} /{ }^{*} p * q}{e^{2}}\right)$, seleccionados aleatoriamente y ubicados o localizados proporcionalmente en las 40 agencias bancarias identificadas anteriormente, según la cantidad de usuarios promedios que acuden a cada agencia. Para la selección de los 384 usuarios, se utilizó el muestreo de números aleatorios, en el cual se eligieron a los usuarios por orden de llegada a la agencia para recibir servicio personal, hasta complementar el tamaño de la muestra de usuarios.

Como técnica de recolección de datos se empleó la técnica de la encuesta personal a los sujetos seleccionados en la muestra, apoyado en dos cuestionarios para recolectar opiniones, actitudes, prácticas de los usuarios sobre el servicio personal prestado en las agencias seleccionadas. El primer cuestionario estuvo destinado a recolectar las expectativas de los clientes y un segundo, las percepciones según el basamento de la escala de Servqual, para así evaluar la calidad de los servicios desde el punto de vista del usuario. Además incluyeron ítems que intentaron caracterizar a los usuarios encuestados (ocupación, grado de instrucción, entre otros).

Ambos instrumentos fueron sometidos a pruebas de validez y confiabilidad. La evidencia sobre la validez de contenido fue obtenida mediante la opinión de expertos. A partir del juicio emitido por un grupo de expertos y el nivel de concordancia entre estos, se calculó el Coeficiente de Proporción de Rangos (Hernández, Fernández, \& Baptista, 2006). También para valorar una propiedad métrica de los instrumentos como lo es la fiabilidad de la escala de Servqual, se utilizó el estadístico Alfa o modelo de consistencia interna de Cronbach. Este modelo, según Pardo y Ruiz (2002), valora la consistencia interna de las escalas unidimensionales a partir de la correlación interelemento promedio, y por ende asegura la consistencia de la escala para medir la misma propiedad u objeto de estudio. Considerando que la escala de Servqual es multidimensional, para la aplicación del estadístico de Alfa dicha escala fue descompuesta en niveles unidimensionales, para ser conformada por un grupo de ítems o declaraciones referidas o encaminadas 
a medir una sola característica, en una misma dirección y con las mismas alternativas de respuestas (escala unidimensional). Por ello se identificaron 10 escalas (cinco referidas a las dimensiones de confiabilidad, responsabilidad, seguridad, empatía y tangibilidad esperada, y otras cinco sobre las mismas dimensiones percibidas por el usuario). Posteriormente, a través del programa SPSS, se obtuvo el número de elementos o ítems analizados en cada escala, así como el valor del coeficiente de confiabilidad Alfa, superior a 0,80 en todas las escalas analizadas ${ }^{2}$, razón por la cual se considera que las escalas utilizadas son fiables, con niveles aceptables y elevados en algunas de estas.

Finalmente y posterior a la recogida de datos, se utilizó la técnica de la estadística inferencial constituida como una prueba paramétrica, conocida como análisis de clases latentes.

\section{Análisis de CLASES LATENTES}

La modelación de una variable latente a partir de una serie de variables observadas depende tanto del tipo o de la métrica observable como de la variable latente. Por ello existen diversas técnicas de modelación con los mismos supuestos y que se diferencian por la métrica de las variables. Actualmente existen ampliaciones de estos modelos que incorporan medidas repetidas y modelos con variables mixtas (continuas y categóricas) (Rivas, 2010).

De acuerdo a Monroy, Vidal y Saade (2010), las vertientes de modelos de agrupamiento latente incluyen modelos factoriales discretos o análisis factorial con clases latentes y modelos de regresión latente o análisis de regresión con clases latentes y análisis de clases latentes. En esta investigación se empleó el análisis de clases latentes (latent class analysis), en el cual se estudia la variabilidad del comportamiento de los individuos de una población, para detectar tipologías, en variables categóricas. En otras palabras, según Pérez y Fajardo (2001), el análisis de clases latentes es una técnica estadística que permite estudiar la existencia de una variable latente (o varias), a partir de un conjunto de variables explicativas observadas y definir, a partir de sus clases, una clasificación o tipología de los individuos 
analizados, en el que tanto las variables observadas como la variable latente deben ser categóricas con dos o más categorías.

El análisis de clases latentes es una técnica clasificatoria utilizada en las ciencias sociales para detectar patrones similares de respuesta y agrupar a los sujetos de una muestra, con características homogéneas, pero diferentes entre sí a los fines de hallar segmentos, clases o grupos; donde la estructura de la asociación de las variables no propone relaciones de causalidad o de contribución sobre una variable dependiente, sino simplemente de asociación (Monroy, Vidal, \& Saade, 2010). De forma más amplia, es una herramienta estadística que permite modelar las relaciones entre las variables observadas, suponiendo que la estructura de relaciones subyacentes es explicada por una variable latente categórica (no observada), las cuales corresponden en esta investigación a diversos grupos de usuarios (segmentos de mercado atendidos).

Esta metodología clasificatoria se basa en la estimación de probabilidades condicionales, lo que permite analizar variables medidas en diferentes métricas categorizadas. Con este análisis estadístico, se comprueba la existencia de diversos grupos de usuarios en las agencias bancarias estudiadas y se infieren las características o patrones de respuesta que permiten distinguir dichos grupos, incluidas sus distintas percepciones de niveles de calidad del servicio.

\section{Resultados}

Para la aplicación del Análisis de Clases Latentes, a los fines de determinar la existencia de distintos grupos de usuarios de los servicios personales de las agencias bancarias del municipio seleccionado e identificar los patrones que permiten distinguirlos, así como para estimar los tamaños de los mismos, se consideraron las siguientes variables categóricas: frecuencia de uso (cantidad promedio de visitas mensuales), otras alternativas de servicio, razones para la selección de la agencia, ocupación, grado de instrucción, tipo de agencia visitada, nivel de calidad y fallas percibidas. Estas variables fueron seleccionadas para el agrupamiento, por considerarse, según Schiffman y Lazar (2001), como aquellas utilizadas 
para segmentar mercados y por su poder para predecir necesidades, gustos, preferencias y comportamiento de los usuarios.

Sobre los niveles de la calidad del servicio, es importante destacar que, la misma fue medida cuantitativamente a partir de la escala de Servqual, como la diferencia entre expectativas y percepciones del usuario se calcularon las puntuaciones de Servqual (Zeithaml et al. 1993a). Cuando dicha puntuación es cercana a cero y positiva, las percepciones del cliente se ajustan a sus expectativas y, por lo tanto, existe calidad; si la puntuación es inferior a cero, las percepciones del cliente respecto al servicio no se ajustan a lo que éste esperaba, por lo cual existe un déficit de calidad. Por el contrario, si la puntuación es superior a cero, existe un derroche o exceso de calidad en el servicio. Por esta razón, la variable nivel de calidad fue categorizada en tres niveles: la categoría déficit de calidad incluyó a todas las puntuaciones menores a cero, la categoría moderada comprendió puntuaciones superiores e iguales a cero e inferiores e iguales a 0,9 , mientras que la categoría de excelente calidad incluyó las superiores pero no iguales a 0,9 .

El Análisis de Clases Latentes se llevó a cabo en dos etapas. En la primera, se consideraron todas las variables, anteriormente señaladas. Así, para identificar el número de clases o segmentos más adecuado, se estimaron modelos de una a cinco clases latentes, cuyos índices de bondad de ajuste se muestran en la Tabla 1. La decisión del número apropiado de clases se basa en los estadísticos AIC (Criterio de Información de Akaike), BIC (Criterio de Información Bayesiana) y en la interpretabilidad del modelo. Cuanto menores sean los valores de los estadísticos, mejor será el modelo porque mayor información contendrá; ambos pueden ser calculados a partir del estadístico $\mathrm{G}^{2}$ (Collins y Lanza, 2010). Ante discrepancia entre los valores de AIC y BIC, se elige este último tomando en consideración que es preferible cuando los tamaños de muestras son grandes. 
Tabla 1

Índices de bondad de ajuste, BIC y AIC, para los modelos de una a cinco clases

\begin{tabular}{|c|c|c|c|c|c|}
\hline & & & Modelo & & \\
\hline & 1 clase & 2 clases & 3 clases & 4 clases & 5 clases \\
\hline AIC & 1517.01 & 1183.36 & 1085.34 & 1067.42 & 1052.53 \\
\hline BIC & 1580.14 & 1313.56 & 1282.61 & 1331.76 & 1383.95 \\
\hline $\mathrm{G}^{2}$ & 1485.01 & 1117.36 & 985.34 & 933.42 & 884.53 \\
\hline
\end{tabular}

Fuente: Elaboración propia a partir de datos obtenidos por el paquete SAS 9.2

El primer modelo a evaluar es el de una clase latente, con el fin de rechazar la hipótesis de independencia, la cual establece si es posible o no dividir a la población en grupos; luego se procede a evaluar la calidad de ajuste de los demás modelos. Un valor alto del estadístico $\mathrm{G}^{2}$, como es el caso, sugiere el rechazo de la hipótesis de independencia, lo cual significa que la población puede dividirse en tantos grupos como clases tenga el modelo. En la Tabla 1, se evidencia que la calidad de ajuste del modelo de tres clases, según el criterio BIC, es la mejor. Esto se reconfirma, al observar cierta estabilización en los valores del índice AIC, a partir de la solución con el mismo número de clases. Sin embargo, también se hizo una inspección de los modelos de dos y cuatro clases latentes, basada en la premisa de que el modelo escogido debía ser el más parsimonioso e interpretable, concluyéndose de dicha evaluación que el modelo de tres clases latentes era el que mejor se ajustaba al conjunto de datos.

Las estimaciones máximo verosímiles de las probabilidades condicionadas y de clases latentes, para el modelo de tres clases, se muestran en la Tabla 2. En esta tabla, las probabilidades condicionales se refieren a la probabilidad de obtener un valor en las variables manifiestas dada la membresía de la clase, donde la suma de las probabilidades de las clases debe ser igual a 1 y cada observación tiene una probabilidad específica de estar en un nivel de la variable observada. Por otra parte, las probabilidades de clases latentes representan el tamaño inferencial para cada uno de los grupos (clases) del modelo. Este tamaño relativo de cada una de las clases la- 
tentes indica cómo se distribuye la población entre el número total de clases el cual, según Rivas (2010), es un parámetro importante para comparar las diferentes poblaciones.

Tabla 2

Probabilidades condicionadas y de clases latentes para el modelo de tres clases

\begin{tabular}{|c|c|c|c|c|}
\hline \multirow{2}{*}{$\begin{array}{l}\text { Variable } \\
\text { independiente }\end{array}$} & \multirow{2}{*}{ Categorías } & \multicolumn{3}{|c|}{ Clases latentes } \\
\hline & & 1 & 2 & 3 \\
\hline \multirow{6}{*}{$\begin{array}{l}\text { Frecuencia de uso } \\
\text { (cantidad promedio } \\
\text { de visitas mensuales) } \\
\text { Otras alternativas de } \\
\text { servicio }\end{array}$} & $<=3$ & 0.2495 & 0.3520 & 0.0038 \\
\hline & $4-5$ & 0.5135 & 0.5045 & 0.0879 \\
\hline & $>=6$ & 0.2370 & 0.1436 & 0.9083 \\
\hline & $\begin{array}{l}\text { Cajeros automáticos e } \\
\text { internet }\end{array}$ & 0.2663 & 0.4637 & 0.0135 \\
\hline & $\begin{array}{l}\text { Ninguna, para el servicio que } \\
\text { demandó }\end{array}$ & 0.3316 & 0.1049 & 0.9099 \\
\hline & Otras & 0.4021 & 0.4314 & 0.0766 \\
\hline \multirow{4}{*}{$\begin{array}{l}\text { Razones para la } \\
\text { selección de la } \\
\text { agencia }\end{array}$} & $\begin{array}{l}\text { Rapidez y buena atención } \\
\text { (eficiencia) }\end{array}$ & 0.0161 & 0.0774 & 0.0002 \\
\hline & $\begin{array}{l}\text { Por su ubicación, } \\
\text { estacionamiento y seguridad }\end{array}$ & 0.2781 & 0.2972 & 0.0467 \\
\hline & $\begin{array}{l}\text { Todas las anteriores } \\
\text { (eficiencia, ubicación, } \\
\text { estacionamiento y seguridad) }\end{array}$ & 0.1976 & 0.2289 & 0.4313 \\
\hline & $\begin{array}{l}\text { Por ser la única que presta el } \\
\text { servicio demandado, aunque } \\
\text { no es la de su preferencia }\end{array}$ & 0.5081 & 0.3964 & 0.5218 \\
\hline \multirow[t]{4}{*}{ Ocupación } & Comerciante o empresario & 0.0032 & 0.5129 & 0.3546 \\
\hline & Estudiante & 0.8442 & 0.0005 & 0.0023 \\
\hline & Otras ocupaciones & 0.0751 & 0.2574 & 0.4732 \\
\hline & $\begin{array}{l}\text { Jubilados, desocupados o } \\
\text { cesantes }\end{array}$ & 0.0775 & 0.2291 & 0.1699 \\
\hline \multirow[t]{3}{*}{ Grado de instrucción } & Básica o media diversificada & 0.9981 & 0.0150 & 0.2132 \\
\hline & Superior técnica & 0.0006 & 0.3233 & 0.2306 \\
\hline & Superior universitaria & 0.0013 & 0.6617 & 0.5562 \\
\hline \multirow{5}{*}{$\begin{array}{l}\text { Tipo de agencia } \\
\text { visitada } \\
\text { Nivel de calidad } \\
\text { percibida }\end{array}$} & Banca comercial & 0.0603 & 0.1409 & 0.0005 \\
\hline & Banca universal & 0.9397 & 0.8591 & 0.9995 \\
\hline & Déficit de calidad & 0.5487 & 0.2910 & 0.4424 \\
\hline & Calidad moderada & 0.4512 & 0.6580 & 0.4689 \\
\hline & Excelente calidad & 0.0001 & 0.0510 & 0.0887 \\
\hline \multirow[t]{3}{*}{ Fallas percibidas } & Sí & 0.8840 & 0.8069 & 0.8344 \\
\hline & No & 0.1160 & 0.1931 & 0.1656 \\
\hline & babilidades de clases latentes & 0.3279 & 0.4724 & 0.1998 \\
\hline
\end{tabular}

Fuente: Elaboración propia a partir de datos obtenidos por el paquete SAS 9.2 
A partir de este análisis inicial, se infiere que la población de usuarios de las agencias bancarias puede ser dividida en tres grupos. El primer grupo muestra las más altas probabilidades de que está conformado por estudiantes, con un nivel de instrucción de educación básica o diversificada concluida, que visitan la agencia de la banca universal entre cuatro y cinco veces al mes por ser la única que presta el servicio, que no poseen otras alternativas del servicio, que perciben fallas y un déficit en la calidad del servicio recibido. Igualmente el segundo y tercer grupo está conformado por empresarios y profesionales de diversas ramas (ingenieros, profesionales independientes y cobradores), con un nivel educativo universitario concluido. Sin embargo, al observar las probabilidades condicionantes de la variable "Tipo de agencia visitada" y "Fallas percibidas", no se observa diferencia significativa entre los grupos o clases conformadas, es decir, todos los grupos de usuarios utilizan las agencias de los bancos universales y perciben fallas, lo cual evidencia que dichas variables no discriminan dadas sus altas probabilidades y poca variabilidad entre clases, para una categoría específica.

En consecuencia, en una segunda etapa, se eliminan las variables "Tipo de agencia visitada" y "Falla percibidas", procediéndose a ejecutar el Análisis de Clases Latentes con el nuevo conjunto de variables. Los índices de bondad de ajuste para los modelos obtenidos con este conjunto de variables, se muestran en la Tabla 3.

Tabla 3

Índices de bondad de ajuste, BIC y AIC, para los modelos de una a cinco clases

\begin{tabular}{|c|c|c|c|c|c|}
\hline & & & Modelo & & \\
\hline & 1 clase & 2 clases & 3 clases & 4 clases & 5 clases \\
\hline AIC & 1087.31 & 754.71 & 667.75 & 679.32 & 693.82 \\
\hline BIC & 1142.54 & 869.12 & 841.35 & 912.10 & 945.78 \\
\hline
\end{tabular}

Fuente: Elaboración propia a partir de datos obtenidos por el paquete SAS 9.2 
Mediante un análisis similar al de la Tabla 1, se seleccionó nuevamente el modelo de tres clases latentes, como el más parsimonioso y de mejor ajuste. Las estimaciones máximo verosímiles de las probabilidades condicionadas y de clases latentes para dicho modelo, se despliegan en la Tabla 4.

Tabla 4

Probabilidades condicionadas y de clases latentes del modelo de 3 clases, excluyendo las variables: "Tipo de agencia visitada" y "Falla percibidas"

\begin{tabular}{|c|c|c|c|c|}
\hline \multirow{2}{*}{$\begin{array}{l}\text { Variable } \\
\text { independiente }\end{array}$} & \multirow{2}{*}{ Categorías } & \multicolumn{3}{|c|}{ Clases latentes } \\
\hline & & 1 & 2 & 3 \\
\hline \multicolumn{2}{|c|}{ Frecuencia de uso $<=3$} & 0.2499 & 0.3506 & 0.0049 \\
\hline \multirow{2}{*}{$\begin{array}{l}\text { (cantidad } \\
\text { promedio de } \\
\text { visitas mensuales) }\end{array}$} & $4-5$ & 0.5099 & 0.5004 & 0.1001 \\
\hline & $>=6$ & 0.2402 & 0.1490 & 0.8951 \\
\hline \multirow{3}{*}{$\begin{array}{l}\text { Otras alternativas } \\
\text { de servicio }\end{array}$} & Cajeros automáticos e internet & 0.2664 & 0.4658 & 0.0071 \\
\hline & $\begin{array}{l}\text { Ninguna, para el servicio que } \\
\text { demandó }\end{array}$ & 0.3320 & 0.1020 & 0.9200 \\
\hline & Otras & 0.4016 & 0.4322 & 0.0729 \\
\hline \multirow{4}{*}{$\begin{array}{l}\text { Razones para la } \\
\text { selección de la } \\
\text { agencia }\end{array}$} & $\begin{array}{l}\text { Rapidez y buena atención } \\
\text { (eficiencia) }\end{array}$ & 0.0162 & 0.0774 & 0.0002 \\
\hline & $\begin{array}{l}\text { Por su ubicación, estacionamiento } \\
\text { y seguridad }\end{array}$ & 0.2787 & 0.2984 & 0.0413 \\
\hline & $\begin{array}{l}\text { Todas las anteriores (eficiencia, } \\
\text { ubicación, estacionamiento y } \\
\text { seguridad) }\end{array}$ & 0.1973 & 0.2283 & 0.4354 \\
\hline & $\begin{array}{l}\text { Por ser la única que presta el } \\
\text { servicio } \\
\text { demandado, aunque no es la de su } \\
\text { preferencia }\end{array}$ & 0.5078 & 0.3959 & 0.5231 \\
\hline \multirow[t]{4}{*}{ Ocupación } & Comerciante o empresario & 0.0038 & 0.5124 & 0.3597 \\
\hline & Estudiante & 0.8381 & 0.0005 & 0.0022 \\
\hline & Otras ocupaciones & 0.0782 & 0.2580 & 0.4708 \\
\hline & Jubilados, desocupados o cesantes & 0.0799 & 0.2291 & 0.1673 \\
\hline \multirow{3}{*}{$\begin{array}{l}\text { Grado de } \\
\text { instrucción }\end{array}$} & Básica o media diversificada & 0.9981 & 0.0135 & 0.2064 \\
\hline & Superior técnica & 0.0006 & 0.3229 & 0.2348 \\
\hline & Superior universitaria & 0.0013 & 0.6636 & 0.5588 \\
\hline \multirow{4}{*}{$\begin{array}{l}\text { Nivel de calidad } \\
\text { percibida }\end{array}$} & Déficit de calidad & 0.5489 & 0.2904 & 0.4413 \\
\hline & Calidad moderada & 0.4510 & 0.6564 & 0.4740 \\
\hline & Excelente calidad & 0.0001 & 0.0531 & 0.0847 \\
\hline & Probabilidades de clases latentes & 0.3303 & 0.4716 & 0.1981 \\
\hline
\end{tabular}

Fuente: Elaboración propia a partir de datos obtenidos por el paquete SAS 9.2 
De la Tabla 4 se resume el perfil de los grupos identificados dentro de la población de usuarios de agencias bancarias en estudio, de la siguiente manera: el primer grupo de usuarios se caracteriza por estar conformado por estudiantes, con un nivel educativo básico o medio diversificado concluido, con una frecuencia moderada de visitas a la agencia (entre cuatro y cinco veces en promedio al mes), con limitadas alternativas de servicio, tales como los cajeros internos, que eligen el servicio de la agencia aunque no es la de su preferencia, y con una percepción de la calidad del servicio deficiente. Esta evaluación de la calidad del servicio, podría estar relacionada con las escasas alternativas del servicio demandado y por los niveles de frecuencia de uso; por cuanto, de acuerdo a Zeithaml et al. (1993a), las expectativas de los usuarios del servicio están determinadas por aspectos asociados a la empatía en cuanto a la accesibilidad del servicio y por sus experiencias anteriores. También debido a las escasas alternativas del servicio y a la elevada frecuencia de uso, se deduce que los usuarios se ven persuadidos a elegir la agencia ("la única que presta el servicio demandado, aunque no es la de su preferencia”), aunque perciban poca calidad en su servicio. Esta primera clase de usuarios agrupa el 33,03\% del total de usuarios de las agencias bancarias.

El segundo grupo, está integrado por empresarios o comerciantes, con un nivel educativo universitario concluido, que visitan a la agencia en promedio entre cuatro y cinco veces al mes, con variadas alternativas de servicio como cajeros internos, automáticos e internet y cajeros automáticos (banca electrónica), y que acuden a la agencia en su mayoría por ser la única que presta el servicio, aunque otras porciones considerables lo hacen por su accesibilidad (ubicación, estacionamiento), seguridad y eficiencia. Esta clase agrupa la mayor parte de usuarios del servicio personal bancario $(47,16 \%$ ) (Tabla 4). Al contrario del grupo anterior, los niveles de calidad con la que dichos usuarios evaluaron los servicios podría ser reflejo de la existencia de variedad de alternativas de servicios para este tipo de usuarios, y estar reflejada en las razones por las que eligió la agencia para recibir el servicio. A su vez la existencia de alternativas de servicio podría ser explicada por el elevado 
grado de instrucción de dichos usuarios (universitario) y por sus ocupaciones, estatus o rol social que impulsan su afiliación a los servicios de la banca electrónica y telefónica.

El tercer grupo está integrado en su mayoría por usuarios de otras ocupaciones, como profesionales independientes, ingenieros, funcionarios públicos y mensajeros o cobradores, con educación universitaria concluida. Estos asisten a las agencias bancarias con elevada frecuencia, en relación a los demás grupos (más de seis veces en promedio al mes), por ser la única que presta el servicio demando, aunque otra porción considerable lo hace por razones, de seguridad, accesibilidad (ubicación y estacionamiento) y eficiencia; además, la mayoría de esta clase de usuarios no tiene otras alternativas para el servicio demandado y califica a la calidad del servicio con niveles aceptables, mientras que otra porción de similar magnitud lo califica con niveles deficientes. Esta última clase agrupa la menor cantidad de usuarios $(19,81 \%)$ de las agencias bancarias (Tabla 4). Al igual que en la primera clase, la inexistencia de alternativas de servicio podría corresponder o estar explicando las leves percepciones de calidad, presionados por la elevada frecuencia de uso del servicio que manifestó la mayoría de usuarios. Dentro de esta clase de usuarios, podría deducirse además que la porción que manifestó haber elegido la agencia por razones de seguridad, accesibilidad y eficiencia, esté reflejada en la porción de usuarios que percibe una calidad aceptable.

\section{CONCLUSIONES Y RECOMENDACIONES}

A través del análisis de clases latentes no hay evidencias suficientes para rechazar las hipótesis planteadas en la investigación y se infiere la existencia de tres grupos de usuarios, ubicados a partir de variables demográficas, psicográficas y conductuales, como el grado de instrucción, la ocupación, percepción de la calidad del servicio, frecuencia de uso, alternativas de servicio y razones para seleccionar la agencia. Estos grupos se extraen a partir de las relaciones o de la asociatividad de características comunes entre los miembros de una misma clase, y la no asociatividad o heterogeneidad de los grupos 
o clases entre sí. El Gráfico 1, resume las características y el tamaño inferido de los grupos de usuarios hallados.

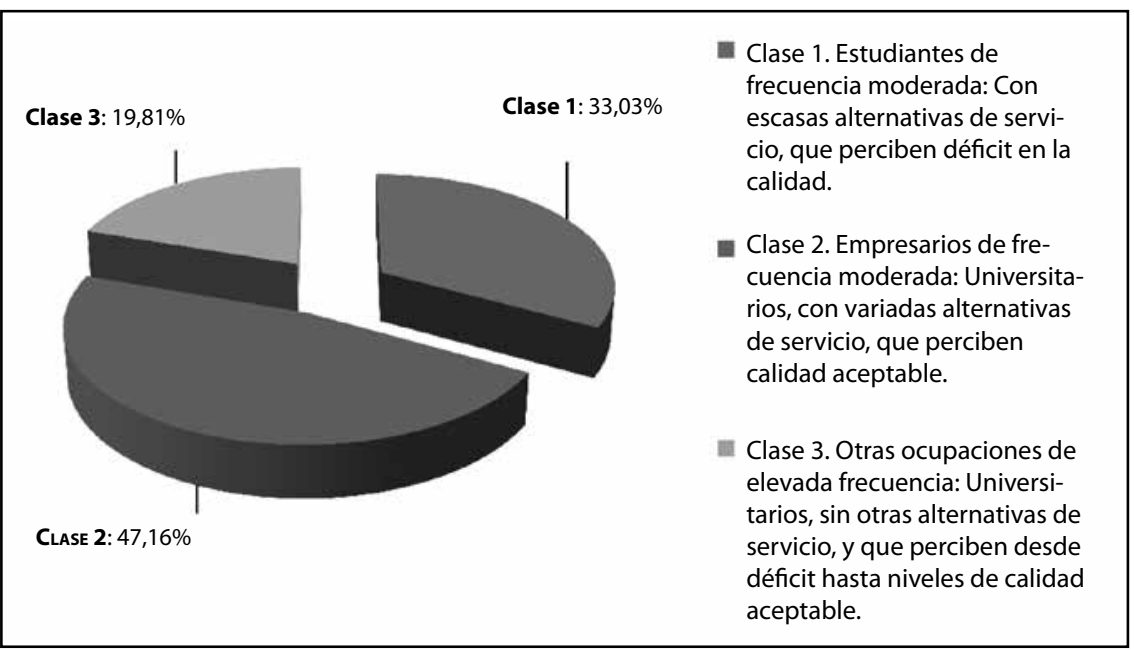

Gráfico 1. Características y tamaños de las clases de usuarios de las agencias bancarias. Elaboración propia a partir de datos obtenidos por el Paquete SAS 9.2.

El análisis de clases latentes, permite identificar y perfilar segmentos de mercados diferenciados, de poca aplicación a los mercados venezolanos, incluidos los financieros; reporta importante información, para la planificación y el posicionamiento de los servicios ofrecidos por las agencias bancarias del municipio Libertador del estado Mérida, Venezuela. Lo anterior es explicado por Kotler y Keller (2006), al afirmar que el posicionamiento y la diferenciación en el mercado deben iniciarse con la identificación de las bases para la segmentación, seguido de la determinación de los perfiles de cada una de las clases o segmentos de usuarios o clientes. Por ello, los resultados arrojados en este trabajo podrían representar las bases para la identificación de segmentos de mercados e iniciar el desarrollo de estrategias adecuadas, previo a la evaluación del atractivo y la rentabilidad de cada uno de estos tres segmentos, para su posterior selección y el posicionamiento de los servicios bancarios en el mercado. Por ejemplo, de llegar a seleccionar el segmento conformado por estudiantes, que perciben déficit en la calidad 
del servicio (Clase 1), previo a la detección y evaluación de su atractivo y rentabilidad reflejado en su perfil de futuros profesionales que los configuran como clientes potenciales de los activos ofrecidos (líneas de crédito, préstamos hipotecarios y demás productos crediticios), se deben establecer medidas para elevar la calidad de servicios ofrecidos. Igualmente, de llegar a comprobarse relación de causalidad entre las variables niveles de calidad deficiente y escasas alternativas de servicio (Tabla 4$)^{3}$, en este segmento de mercado deben ampliarse las alternativas de servicio mediante la oferta de autoservicios (cajeros automáticos) y banca telefónica, entre otras, aprovechando el potencial de aprendizaje y desaprendizaje de los jóvenes estudiantes, proclives a los cambios y a los producto nuevos o mejorados. Esta introducción de alternativas de servicio es una medida para incrementar la calidad del servicio, expresado en la empatía como dimensión importante.

Igualmente, al reflexionar que dentro de los tres grupos de usuarios hallados, la calidad no es calificada como excelente por una porción importante de usuarios (Tabla 4), sino que la mayoría de los usuarios de todas las clases percibieron fallas (Tabla 2), es, por consiguiente, recomendable en las agencias bancarias, diseñar prácticas que permitan elevar la calidad del servicio personal y recuperar el servicio, y más aún cuando la mayor parte de los usuarios de todos los grupos manifestó haber seleccionado la agencia por ser la única que presta el servicio demandado aunque no es la de su preferencia. Incluso, deben continuar satisfaciendo y manteniendo la calidad del servicio prestado a las clases de usuarios que manifestaron niveles de calidad aceptables (clases 2 y 3 ), para mantener relaciones favorables con dichos usuarios e incentivar las comunicaciones boca a boca de manera positiva.

Para elevar la calidad de los servicios, existe un amplio abanico de estrategias con diversos enfoques, desde la recuperación del servicio, pasando por modelos específicos que analizan factores o dimensiones que inciden en la calidad del servicio. En cuanto a la recuperación del servicio, estas son todas las respuestas del proveedor ante una falla, error u omisión, para lo cual se deben identificar y resolver las quejas y aprender de las experiencias. Entre las acciones efectivas 